\title{
Formulation and In-Vitro Evaluation of Piroxicam Loaded BSA Nanospheres by Desolvation
}

\section{Sravya Neela and Kiran Babu Uppuluri*}

Bioprospecting Laboratory, School of Chemical and Biotechnology, SASTRA University, Thanjavur- 613 401, Tamil Nadu, India

\begin{abstract}
Protein nanoparticles offer improved delivery of drugs particularly targeted delivery to specific cells by ligand attachment, sustained and triggered release. Non steroidal anti-inflammatory drug (NSAID), Piroxicam (PRX) is one of the most widely used drug for treating pain and inflammation. But despite its potent analgesic action it has few draw backs like low absorption rate due to poor water solubility and side effects due to non specific inhibition of Cyclooxygenase enzymes. In this context here, we report a new PRX self-assembled nano sphere delivery system developed from bovine serum albumin (BSA) using a very simple, rapid and reliable method, desolvation. Synthesized nanoparticles were characterized by particle size distribution, zeta potential, polydispersity index, FTIR, scanning electron microscopy and in-vitro release studies. The formulated BSA-PRX nano particles exhibited a uniform spherical shape with an average size of $388.7 \mathrm{~nm}$. In vitro release behavior of the drug from BSA conjugate suggests that about $50 \%$ of the drug was released during first $3 \mathrm{~h}$ and $85 \%$ after $18 \mathrm{~h}$.
\end{abstract}

Keywords: Piroxicam (PRX); Bovine serum albumin (BSA); Nanospheres; In-vitro release

\section{Introduction}

Piroxicam (PRX) is a non-steroidal anti-inflammatory drug that is most widely used in the treatment of rheumatic conditions like inflammation, pain due to injury, menstrual cramps, arthritis, and other musculoskeletal conditions and non-rheumatic conditions like biliary and ureteric colic, dysmenorrheal inflammation and fever [1]. PRX acts by inhibiting two cyclooxygenase isozymes (COX-1 and COX-2) which catalysis the prostaglandins (PG) synthesis. PG mediates many physiological functions of the body, including control of blood pressure; control of respiratory and gastrointestinal tract smooth muscles [2]. COX-1 is involved in maintaining the physiological function of the gastrointestinal and renal tracts where as COX-2 participates in the pain and inflammation [3]. Anti-inflammatory actions of PRX are due to the inhibition of COX-2 only whereas the inhibition of COX-1 leads to the unwanted side-effects like dyspepsia, heartburn, nausea and vomiting [4]. In fact a very low oral bioavaibility of PRX was observed due to its poor water solubility nature. Hence, there is a considerable interest in developing new formulations of PRX to improve its oral absorption rate as well as to target COX-1specific [5].

Nanotechnology has given a path breaking directions to pharmaceutical formulations. One of the major features is the synthesis of drug delivery systems at nano size, this permits the drug molecule easy accessibility into typical anatomical sites by escaping phagocytosis and entering tiny capillaries. Formulation of nanometer sized drug particles with large surface area and stabilized by polymers arises as an attractive and efficient approach to enhance the dissolution rate of poorly soluble drugs [6,7]. In this regard biopolymers are more attractive as they proven to be biocompatible, biodegradable, non toxic nature and non antigenecity [8]. In addition these polymers can effectively deliver the drug to target site therefore increasing the therapeutic benefit and minimizes the side effects $[1,9,10]$.

Recently, protein nanoparticles (NPs) especially albumin nano particles have drawn significant attention in the drug delivery as a novel drug carrier due to their high drug binding capacity and negligible side effects [11-14]. A wide variety of compounds can be entrapped using albumin as it carries charged amino acid moieties which allow the electrostatic adsorption of positively and negatively charged molecules to its surface. In the blood albumin plays a major role for transporting macromolecules to the target organs; perhaps this also makes the albumin a potential macromolecular carrier for the target drug delivery systems [14].

Desolvation technique for amphiphilic macromolecular cross linking is commonly used for the preparation of protein drug NPs [15]. Desolvation is a thermodynamically driven self-assembly process by which drugs get encapsulated into polymers yielding nano sized particles [16]. The size of the particle depends on the preparation conditions like protein content, $\mathrm{pH}$, ionic strength, concentration of cross- linking agent, agitation speed and amount of desolvating agent [17]. Cross-linking is the major step in the desolvation step and involves three stages: protein dissolution, protein aggregation and protein deaggregation. Alcohol, the desolvation agent maintains the aggregate size and glutaraldehyde makes the cross linkage. Desolvation technique only can give nanospheres as final product as it deaggregates protein colloidal suspension into milky white appearance. Both hydrophilic and hydrophobic drugs can be encapsulated in to nano spheres or nano particles using this technique $[18,19]$.

The prominent aim of this research was to study the entrapment phenomenon of a poorly soluble drug PRX into bovine serum albumin (BSA) and formulating into nano spheres by a very simple desolvation technique. Therefore, BSA-PRX NPS will not selectively target COX2 and avoids adverse reactions. The physico chemical parameters and morphology of the BSA loaded PRX were characterized. The interaction between BSA and PRX was studied by using FT-IR spectroscopy. Particle

*Corresponding author: Kiran Babu Uppuluri, Bioprospecting Laboratory, School of Chemical and Biotechnology, SASTRA University, Thanjavur- 613401 , Tamil Nadu, India, Tel: 91-4362-264101-3635; Fax: 91-4362-264120; E-mail: kiranbabu@scbt.sastra.edu

Received March 05, 2015; Accepted April 20, 2015; Published May 10, 2015

Citation: Neela S, Uppuluri K (2015) Formulation and In-Vitro Evaluation of Piroxicam Loaded BSA Nanospheres by Desolvation. J Nanomed Nanotechnol 6: 289. doi: $10.4172 / 2157-7439.1000289$

Copyright: @ 2015 Neela S, et al. This is an open-access article distributed unde the terms of the Creative Commons Attribution License, which permits unrestricted use, distribution, and reproduction in any medium, provided the original author and source are credited. 
size, polydispersity index (PDl) and zeta potential of nanospheres were evaluated by photon correlation spectroscopy (PCS). The scanning electron microscopy (SEM) was used to study the morphology of microspheres. The entrapment efficiency of PRX in nanospheres was assessed and the In-vitro release profile was evaluated.

\section{Materials and Methods}

\section{Chemicals}

Piroxicam (PRX), fatty acid free bovine serum albumin (BSA), ethanol and glutaraldehyde were purchased from Sigma Aldrich, India. All of other chemicals and reagents of analytical grade were obtained from Merck India.

\section{Preparation of BSA PRX nanoparticles}

PRX loaded BSA nanoparticles were prepared by ethanol desolvation method according to Marty et al 1978 [20]. Briefly, in 2 $\mathrm{mL}$ Phosphate buffer ( $\mathrm{pH} 7.4)$, PRX (20 mg) and BSA (200 mg) were incubated overnight at $37^{\circ} \mathrm{C}$. Then the $\mathrm{pH}$ of the solution was adjusted to 8.0 with $1 \mathrm{~N} \mathrm{NaOH}$ and was stirred for 5 minutes. This aqueous phase was desolvated by drop wise addition of ethanol at constant stirring till the solution turns turbid and opalescent. Glutaraldehyde solution (100 $\mu \mathrm{l}$ of the $8 \%$ ) was used to cross link and stabilize the BSA loaded PRX NPs. The resultant solution was kept for stirring overnight, centrifuged at $15000 \mathrm{rpm}$; the pellet was collected, lyophilized and stored at $4^{\circ} \mathrm{C}$ [21,22].

\section{Characterization of PRX loaded BSA nano particles}

The size and the zeta potential of BSA-PRX NPs were measured by Photon Correlation Spectroscopy (PCS) using a Malvern Zeta sizer $3000 \mathrm{HS}$ with He-Ne laser $(633 \mathrm{~nm})$ at a scattering angle of $90^{\circ} \mathrm{C}$. The interaction between BSA and PRX was studied by using FTIR spectroscopy using potassium bromide $(\mathrm{KBr})$ pellet technique. In which, samples were mixed in a mortar with $\mathrm{KBr}(1: 100)$ and pressed in a hydraulic press ( 9 tons) to small tablets and then analyzed by transmittance technique with $4 \mathrm{~cm}^{-1}$ resolution. The spectra of free BSA, pure PRX, BSA-PRX suspension and BSA-PRX NPs were recorded on a Perkin Elmer FT-IR spectrometer (Perkin Elmer, India) in a range of about $400-4000 \mathrm{~cm}^{-1}$. The morphology and size of the PRX loaded BSA nanoparticles was studied by Scanning Electron Microscopy (SEM) (JEOL JSM 670F-6701) at a voltage of around $3 \mathrm{keV}$.

\section{Spectroscopic determination of Piroxicam}

Quantitative determination of PRX was determined by visible spectroscopy according to Azmi et al 2009 [23]. The stock solution of PRX $(1.6 \mathrm{mg} / \mathrm{ml})$ was prepared in ethanol and stored at $4^{\circ} \mathrm{C}$. To the aliquots stock solutions of PRX $(0.05$ to $1.0 \mathrm{~mL}) 1.0 \mathrm{~mL}$ of $0.005 \mathrm{M}$ ferric sulphate solution was added and diluted up to $10 \mathrm{~mL}$ with ethanol. Absorbance was measured at $504 \mathrm{~nm}$ using Thermo Evolution $201 \mathrm{UV}$ visible spectrophotometer. Calibration graph was plotted, regression equation was fitted and correlation coefficient value (R) was calculated.

\section{Entrapment efficiency of Piroxicam}

The amount of PRX entrapped into BSA-PRX nanospheres was measured by visible spectrophotometry at $504 \mathrm{~nm}$. The entrapment efficiency of PRX was determined as follow:

$$
\operatorname{PRX}_{\text {Entrapment }}(\%)=\frac{[\mathrm{PRX}]_{\text {initial }}-[\mathrm{PRX}]_{\text {final }}}{\left[\mathrm{PRX}_{\text {initial }}\right]} \times 100
$$

Where $[\mathrm{PRX}]_{\text {initial }}$ is the initial concentration of Piroxicam used and $[\mathrm{PRX}]_{\text {final }}$ is the final concentration of Piroxicam in supernatant. Measurements were recorded in triplicate and the mean value \pm standard deviation was reported.

\section{In- vitro drug release studies}

Lyophilized nano particles were accurately weighed and dispersed to the original volume in phosphate buffer $\mathrm{pH}$ 7.4. Release pattern of PRX from BSA-PRX NPs was observed by using Dissolution apparatus USP type 1 (Basket). It consists of a cellophane tube tied with a dialysis membrane that kept in basket type apparatus and the basket contains PBS ( $\mathrm{pH}$ 7.4). The BSA-PRX NPs were placed inside the dialysis membrane and drug release studies were carried at room temperature. The samples were withdrawn periodically and measured for PRX concentration. The volume of dissolution medium was kept constant by the addition of PBS for every sample collection. The same procedure was followed for both drug and formulation for about $36 \mathrm{~h}$. The experiments were carried in triplicate and results are reported as the mean \pm standard deviation.

The mechanism of the release pattern of the active ingredient from the formulation was analyzed by mathematical modeling. Dissolution data was investigated with models including zero order, first order, Higuchi, Korsemeyer-Peppas, Hixson-Crowell, Hopfenberg, Baker Lonsdale, Makoid-Banakar, Weibull and Gompertz using the software DD solver [21].

\section{Results and Discussion}

Though Silva et al., reported the preparation and characterization of BSA-PRX and HAS- PRX microspheres by sonochemical method [1] and other researchers [24-26] demonstrated the feasibility of synthesizing PRX NPS but still there is a scope for the development of fast, effective and reliable NPs to effectively deliver the PRX at the site of action. Therefore in this present work the formulation and evaluation of BSA entrapped PRX nanospheres was demonstrated using a very simple and rapid method, desolvation in order to achieve therapeutic benefits like enhancing the absorption and site specificity of PRX.

\section{Preparation and characterization of BSA PRX NPs}

The BSA PRX NPs were prepared by desolvation method and cross linked with glutaraldehyde. The average hydrodynamic size of PRXloaded BSA NPs was $388.7 \mathrm{~nm}$ (Figure 1A). This was larger than the size of $27.9 \mathrm{~nm}$ measured by SEM, which may be due to the shrinkage of the particles during dehydration. The poly dispersity index (pdi) was found to be 0.419 , indicating the presence of different sized nanoparticles, which is consistent with SEM micrographs. The difference between SEM and particle size analyzer is consistent with the previous reports. The particle size and surface charge of NPs is very important for their recognition or non recognition by the defense mechanism of body. Therefore, we measured the zeta potential of the BSA-PRX NPs (Figure 1B). Zeta potential value obtained for BSA NPs and BSA-PRX NPs are -15.4 and $-26.5 \mathrm{mV}$, respectively. The negative sign indicates the alkaline nature of the $\mathrm{pH}$ and negative charges on the nano spheres. The potential difference suggested an increased stability of BSA NPs due to the interaction between PRX and BSA. The stabilty of the collioid system is mostly depends on the negatively charged particles and prevention of coulombic repulsive forces between the particles from agglomerating in the colloid state [21,27]. A minimum zeta potential value of $-20 \mathrm{mV}$ is highly desirable in case of combined electrostatic and steric stabilization of nanoparticles [28].

FTIR spectra of pure PRX, BSA and BSA-PRX and BSA-PRX NPs 

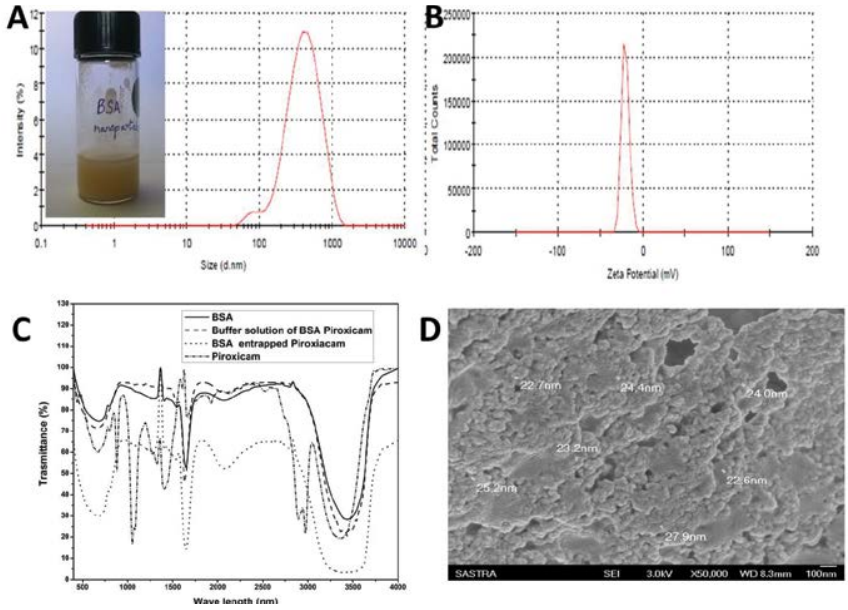

Figure 1: (A) Particle size distribution (inset, appearance of BSA-PRX NPs) and (B) zeta potential of BSA-PRX NPs (C) FTIR spectra of bulk PRX, BSA, BSA-PRX suspension and BSA-PRX NPS (D) Scanning electron micrograph of BSA-PRX NPs.

are shown in Figure 1C. In the NPs spectrum, the N-H stretching can be found at $3462 \mathrm{~cm}^{-1}$, the $\mathrm{C}=\mathrm{O}$ stretching at $1660 \mathrm{~cm}^{-1}$, the $\mathrm{OH}$ bending at $1631 \mathrm{~cm}^{-1}$, the $\mathrm{C}=\mathrm{N}$ stretching at $1530 \mathrm{~cm}^{-1}$, the $\mathrm{SO} 2$ asymmetric stretching at 1334 and $1365 \mathrm{~cm}^{-1}$ and the $\mathrm{SO} 2$ symmetric stretching at $1174 \mathrm{~cm}^{-1}$. While the $\mathrm{NH}$ stretching in bulk PRX and in coarse suspension is at $3402 \mathrm{~cm}^{-1}$, in the nanosuspension is at $3462 \mathrm{~cm}$ ${ }^{1}$, the $\mathrm{C}=\mathrm{O}$ stretching shifted from 1681 to $1660 \mathrm{~cm}^{-1}$. The spectrum of PRX showed strong absorption of $-\mathrm{C}=\mathrm{O}$ and $-\mathrm{CONH}$ between 1550 $-1750 \mathrm{~cm}^{-1}$. BSA shows -CONH- streching frequency between 1600 $-1700 \mathrm{~cm}^{-1}$. The spectra of BSA-PRX NPs showed a significant shift in the a characterisitic carbonyl $\left(1661 \mathrm{~cm}^{-1}\right)$ streching frequency region, suggesting an interaction between the protein and drug.

SEM of BSA-PRX NPs is shown in Figure 1D, which appeared as spherical in shape with smooth surfaces and nearly uniform in size. The size of the NPs indicated by SEM was about $30 \mathrm{~nm}$. In general the spherical shape of the nanoparticles maintains minimum contact with the aqueous environment and offers the longest diffusion pathways due to small specific area thus the highest potential for controlled release of encapsulated drugs [14].

\section{Entrapment efficiency of Piroxicam}

Entrapment efficiency is an important factor that reflects the amount of drug that entrapped in nano spheres in relation to the total amount of drug used [1]. At the chosen experimental condition, the encapsulation efficiency of PRX within BSA NPs was cacluated to be $94.27 \pm 0.62 \%$. The \% leakage of PRX due to colaescence and break up of emulsion droplets and drug diffusion from aqueous phase to oil phase was investigated upon storage at $4^{\circ} \mathrm{C}$ for 2,4 and 8 weeks. The leakage was found to be less than $1 \%$ with neither alteration in physical appearance nor aggregation of the NPs.

\section{In- vitro drug Release studies}

For class II drugs (poor solubility, high permeability) like PRX, dissolution rate very often controls the rate of oral absorption [29]. Both PRX and BSA-PRX NPs dissolution tests were studied and bulk PRX dissolution rate was compared with those of BSA-PRX NPs and the results depicted in Figure 2. From the figure, a rapid release of PRX at initial stages was observed in both pure and nano formulation. This may be due to the desorption and diffusion of PRX from the outer surface of nano particles $[21,30]$. It is always prefeered to have intial burst from the controlled drug delivery systems to attain threshold dose and elicit therapeutic action. After the initial rapid release BSA-PRX NPs shown a slow and sustained release continuously and the maximum drug was release in $30 \mathrm{~h}$. Whereas the pure drug had shown the initial burst release and was shown the maximum amount of drug release within $3 \mathrm{~h}$. The slow release pattern of nanoformulation after initial rapid release has been reported earlier and our system is consistent with literature and shows slow diffusion of PRX across the albumin matrix of the NPs $[5,14,19,21,30]$. To maintain the therapuetic safe concentrations for a stipulated time and to avoid the systemic toxic effects, always a slow and sustained release of drug form the formulation is required and highly desirable. Therefore, we suggest that the BSA-PRX nano formulations will offer an effective controlled delivery system with reduced systemic side effects and may also enhance the targeting efficiency to deliver drug into the target organ.

In-vitro drug release data was found to best fit Korsmeyer-Peppas

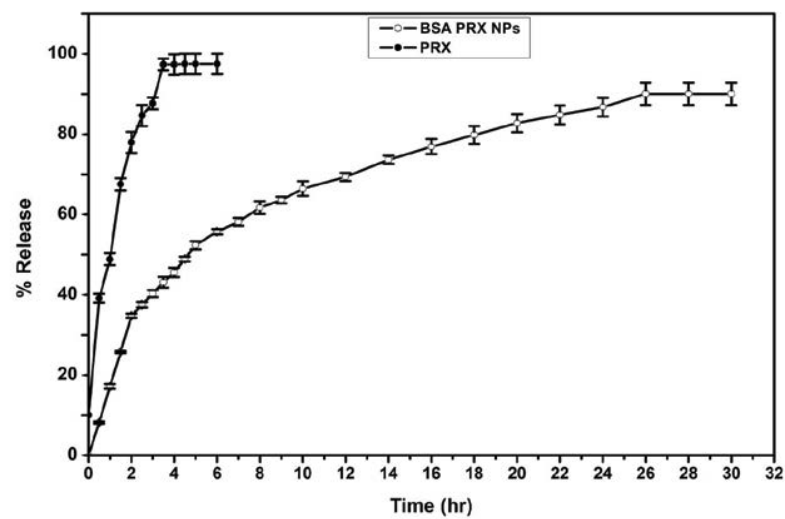

Figure 2: In vitro drug release profile for bulk PRX and BSA-PRX NPS.

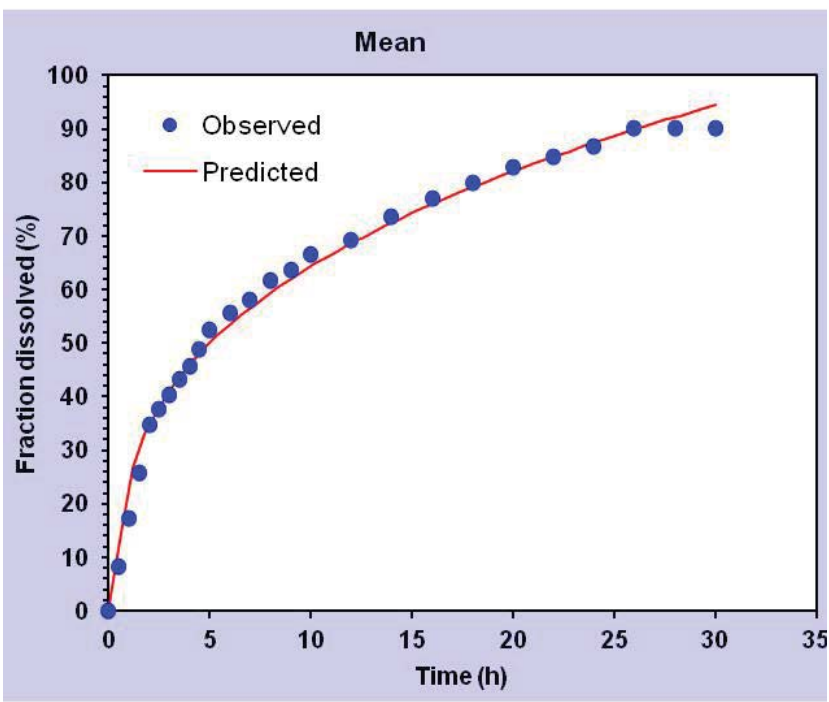

Figure 3: Experimental PRX release Vs predicted PRX release. 
Citation: Neela S, Uppuluri K (2015) Formulation and In-Vitro Evaluation of Piroxicam Loaded BSA Nanospheres by Desolvation. J Nanomed Nanotechnol 6: 289. doi:10.4172/2157-7439.1000289

with Tlag Model. It was confirmed with obtained $\mathrm{R}^{2}$ value $>0.99$, suggesting diffusion as the principal mechanism of drug release. The Pareto plot drawn with experimental and predicted values showed a satisfactory correlation between both (Figure 3).

\section{Conclusion}

Developing protein nanoparticles as drug delivery devices emerged as a promising and efficient method for formulating various classes of drugs due to their biocompatibility, biodegradability and the slow release of drug in blood. In the present work, BSA-PRX nanospheres were successfully formulated by a very simple, fast and reliable method, desolvation process. The FTIR studies were performed for drug, protein, nano formulation, and for drug- protein sample and it was found that drug was entrapped in the protein NPs. In-vitro release profiles of free drug and nanoparticle suspension confirms that this colloidal drug carrier is capable of releasing drug in a controlled manner. The nanospheres have shown high stability in repetitive experiments carried up to 6 months. However, further studies involving In-vivo experiments are required to consider it as a drug delivery system on a commercial scale.

\section{References}

1. Silva R, Ferreira H, Carvalho AC, Gomes AC, Cavaco-Paulo A (2012) Protein microspheres as suitable devices for piroxicam release. Colloids Surf B Biointerfaces 92: 277-285.

2. Simmons DL, Botting RM, Hla T (2004) Cyclooxygenase isozymes: the biology of prostaglandin synthesis and inhibition. Pharmacol Rev 56: 387-437.

3. Frölich JC (1995) Prostaglandin endoperoxide synthetase isoenzymes: the clinical relevance of selective inhibition. Ann Rheum Dis 54: 942-943.

4. Brooks $P$ (1998) Use and benefits of nonsteroidal anti-inflammatory drugs. Am J Med 104: 9S-13S.

5. Lai F, Pini E, Angioni G, Manca ML, Perricci J, et al. (2011) Nanocrystals as too to improve piroxicam dissolution rate in novel orally disintegrating tablets. Eur $J$ Pharm Biopharm 79: 552-558.

6. Keck CM, Müller RH (2006) Drug nanocrystals of poorly soluble drugs produced by high pressure homogenisation. Eur J Pharm Biopharm 62: 3-16.

7. Merisko-Liversidge E, Liversidge GG, Cooper ER (2003) Nanosizing: a formulation approach for poorly-water-soluble compounds. Eur J Pharm Sci 18: $113-120$

8. Doshi N, Mitragotri S (2009) Designer biomaterials for nanomedicine. Advanced Functional Materials 19: 3843-3854.

9. Won YW, Kim YH (2008) Recombinant human gelatin nanoparticles as a protein drug carrier. J Control Release 127: 154-161.

10. Couvreur P, Gref R, Andrieux K, Malvy C (2006) Nanotechnologies for drug delivery: Application to cancer and autoimmune diseases. Progress in solid state chemistry 34: 231-235.

11. Merodio M, Arnedo A, Renedo MJ, Irache JM (2001) Ganciclovir-loaded albumin nanoparticles: characterization and in vitro release properties. Eur J Pharm Sci 12: 251-259.

12. Brigger I, Dubernet $C$, Couvreur $P$ (2002) Nanoparticles in cancer therapy and diagnosis. Adv Drug Deliv Rev 54: 631-651.
13. Jiang W, Kim BY, Rutka JT, Chan WC (2007) Advances and challenges of nanotechnology-based drug delivery systems. Expert Opin Drug Deliv 4: 621633.

14. Sripriyalakshmi S, Anjali CH, George PD, Rajith B, Ravindran A (2014) BSA nanoparticle loaded atorvastatin calcium--a new facet for an old drug. PLoS One 9: e86317.

15. Azarmi S, Tao X, Chen H, Wang Z, Finlay WH, et al. (2006) Formulation and cytotoxicity of doxorubicin nanoparticles carried by dry powder aerosol particles. Int J Pharm 319: 155-161.

16. Weber C, Coester C, Kreuter J, Langer K (2000) Desolvation process and surface characterisation of protein nanoparticles. Int J Pharm 194: 91-102.

17. Coester CJ, Langer K, van Briesen H, Kreuter J (2000) Gelatin nanoparticles by two step desolvation--a new preparation method, surface modifications and cell uptake. J Microencapsul 17: 187-193.

18. Sripriyalakshmi S, Jose P, Ravindran A, Anjali CH (2014) Recent trends in drug delivery system using protein nanoparticles. Cell Biochem Biophys 70: 17-26.

19. Jun JY, Nguyen HH, Paik SYR, Chun HS, Kang BC, et al. (2011) Preparation of size-controlled bovine serum albumin (BSA) nanoparticles by a modified desolvation method. Food Chemistry 127: 1892-1898.

20. Marty JJ, Oppenheim RC, Speiser P (1978) Nanoparticles--a new colloidal drug delivery system. Pharm Acta Helv 53: 17-23.

21. Wilson B, Lavanya Y, Priyadarshini SR, Ramasamy M, Jenita JL (2014) Albumin nanoparticles for the delivery of gabapentin: preparation, characterization and pharmacodynamic studies. Int J Pharm 473: 73-79.

22. Maghsoudi A, Shojaosadati SA, Vasheghani Farahani E (2008) 5-Fluorouracilloaded BSA nanoparticles: formulation optimization and in vitro release study. AAPS PharmSciTech 9: 1092-1096.

23. Azmi SNH, Iqbal B, Jaboob MAM, Al Shahari WAS, Rahman N (2009) Spectrophotometric Determination of Piroxicam via Chelation with Fe (III) in Commercial Dosage Forms. Journal of the Chinese Chemical Society 56 : 1083-1091.

24. Otarola J, Lista AG, Band BF, Garrido M (2014) Capillary electrophoresis to determine entrapment efficiency of a nanostructured lipid carrier loaded with piroxicam. Journal of Pharmaceutical Analysis.

25. Javadzadeh $Y$, Bairami Atashgah $R$, Barzegar-Jalali $M$, Soleimani $F$ Mohammadi G, et al. (2014) Inclusion of piroxicam in mesoporous phosphate glass-ceramic and evaluation of the physiochemical characteristics. Colloids Surf B Biointerfaces 116: 751-756.

26. Adibkia K, Shadbad MRS, Nokhodchi A, Javadzedeh A, Barzegar-Jalali M et al. (2007) Piroxicam nanoparticles for ocular delivery: physicochemical characterization and implementation in endotoxin-induced uveitis. J Drug Target 15: 407-416.

27. Mainardes RM, Evangelista RC (2005) PLGA nanoparticles containing praziquantel: effect of formulation variables on size distribution. Int J Pharm 290: 137-144.

28. Kohane DS, Tse JY, Yeo Y, Padera R, Shubina M, et al. (2006) Biodegradable polymeric microspheres and nanospheres for drug delivery in the peritoneum. J Biomed Mater Res A 77: 351-361.

29. Tantishaiyakul V, Kaewnopparat N, Ingkatawornwong S (1999) Properties of solid dispersions of piroxicam in polyvinylpyrrolidone. Int J Pharm 181: 143151.

30. Xie G, Zhang G, Lin F, Zhang J, Liu Z, et al (2008) The fabrication of subwavelength anti-reflective nanostructures using a bio-template. Nanotechnology 19: 095605. 